\title{
Key immune system markers in immunotherapy: absolute lymphocyte count, neutrophil to lymphocyte ratio, and alternative immune system metrics
}

\author{
Victor E. Chen, Benjamin A. Greenberger, James M. Taylor, Bo Lu \\ Department of Radiation Oncology, Sidney Kimmel Medical College \& Cancer Center at Thomas Jefferson University, Philadelphia, PA, USA \\ Correspondence to: Bo Lu, MD, PhD. Department of Radiation Oncology, Sidney Kimmel Medical College \& Cancer Center at Thomas Jefferson \\ University, 111 South $11^{\text {th }}$ Street, G-301 Bodine Cancer Center, Philadelphia, PA 19107, USA. Email: Bo.Lu@jefferson.edu. \\ Comment on: Pike LRG, Bang A, Mahal BA, et al. The Impact of Radiation Therapy on Lymphocyte Count and Survival in Metastatic Cancer \\ Patients Receiving PD-1 Immune Checkpoint Inhibitors. Int J Radiat Oncol Biol Phys 2019;103:142-51.
}

Received: 24 August 2019; Accepted: 05 September 2019; Published: 17 September 2019.

doi: $10.21037 /$ tro.2019.09.01

View this article at: http://dx.doi.org/10.21037/tro.2019.09.01

Immunotherapy is an increasingly popular therapeutic strategy in modern cancer care, with significant research and development invested in optimizing its application in combination with existing modalities, including radiation therapy. A recent article in the International fournal of Radiation Oncology, Biology, Physics by Pike et al. explores the intricate relationship between radiotherapy (RT), lymphocyte count, immune checkpoint inhibition (ICI) and clinical outcomes (1). In this study, the authors attempt to address an evolving question: given conflicting data suggesting that RT may either enhance or diminish the effectiveness of ICI (through competing pathways such as the immunogenic release of antigen and cytokines, T-cell receptor diversification, and decreased lymphocyte count), what is the impact on patients with metastatic disease who are treated with both modalities?

As this article provides an appropriate framework with which to evaluate the current landscape of immunotherapy research with regards to relevant prognostic laboratory markers, a brief overview of the study is provided here. In this study, a multi-center retrospective review was conducted of patients with metastatic non-small cell lung cancer, melanoma, and renal cell carcinoma who received both palliative RT and ICI with nivolumab and/or pembrolizumab as part of their multidisciplinary oncologic care (1). They use absolute lymphocyte count (ALC) and neutrophil to lymphocyte ratio (NLR) as surrogates to assess the impact of radiation therapy on lymphocyte count, defining severe lymphopenia as ALC $<500$. In order to isolate the effect of and interplay between ICI and RT, the authors exclude patients who received cytotoxic chemotherapy concurrent with RT or ICI, or while cell counts were being measured. In total, 110 patients were included in the study and 225 courses of palliative radiotherapy were delivered, with a median observed change in ALC after RT of -161 cells $/ \mathrm{mL}$. This decrease was found to be greater with RT to the spine, lung/mediastinum and chest wall as compared with the brain, extremity and abdomen/pelvis, as well as with courses of RT that lasted longer than five fractions. The authors demonstrate that patients who develop radiation-induced severe lymphopenia were more likely to have severe lymphopenia at initiation of ICI, which itself is associated with increased mortality on multivariable analysis. Given this, Pike et al. propose that extracranial and prolonged courses of RT increase the risk of severe lymphopenia, which is associated with poorer survival in patients treated with ICI (1).

As much of the current literature that seeks to describe how immune-related laboratory markers change in response to various cancer treatment modalities, and the resulting impact on prognosis, has focused on metrics relating to the lymphocyte cell line, the paper by Pike et al. serves as an appropriate lens with which to evaluate research trends today and propose future directions in prognostic immune system metrics and immunotherapy. For example, Grossman et al. noted how standard of care therapy for high-grade gliomas typically involves a combination of radiotherapy, temozolomide, and glucocorticoids, and that each of these 
treatments is known to independently suppress immune system function (2). Their work was partially inspired by an earlier study that demonstrated CD4-specific lymphopenia, and the subsequent development of opportunistic infections, in patients with melanoma who were treated with temozolomide (3). Therefore, they investigated CD4 lymphocyte count as a measure of immune system function, and found that significant decreases in this value occurred over the course of standard-of-care treatment. In this patient population of nearly one hundred patients-of which the vast majority had the diagnosis of glioblastoma-the CD4 count decreased from a median of 664 per cubic millimeter prior to initial treatment, to 255 per cubic millimeter two months after starting radiation and temozolomide. Interestingly, the authors noted minimal recovery in CD4 counts and suggest that patients who have severely low CD4 counts of under 200 per cubic millimeter due to adverse effects from treatment are at higher risk of early death from tumor progression (2). In a later publication, Grossman et al. examined multiple different solid tumor histologies to determine if lymphopenia attributable to chemoradiation could be found in other disease types (4). They found that over forty percent of a cohort of patients with diagnoses of either pancreatic cancer, non-small cell lung cancer, and malignant glioma developed new, severe, and persistent lymphopenia approximately two months after starting treatment; as lymphopenia was again found to be associated with shorter survival, they argued that further research should be conducted with the aim of preventing or reversing this condition.

Aside from levels of lymphocytes in the entire peripheral bloodstream, investigators have also focused specifically on tumor-infiltrating lymphocyte metrics, as immune system functionality in the immediate tumor microenvironment likely more directly influences disease control in the local setting. Fukunaga et al. demonstrated that in surgically resected specimens of pancreatic adenocarcinoma, the presence of both CD4 and CD8 lymphocytes (defined as greater than twenty CD4 cells and greater than one hundred CD8 cells, per five fields examined at 400x magnification) in the resected tumor sample was statistically significant for increased survival (5). Neither CD4 nor CD8 presence alone, however, remained significant on multivariate analysis. Based on these findings, the authors suggest that cooperation between both major lymphocyte cell lines is critical for adaptability of the host immune system and a robust immune system response.

Fortunately, initial efforts at identifying how to adjust traditional treatment paradigms for this relatively newfound importance on lymphocyte count have begun. For instance, Li et al. describe that in the setting of administering stereotactic radiosurgery (SRS) for brain metastases from primary lung cancer, in contrast to the expected stimulation of anti-tumor host immunity, patients with lymphopenia, as measured by ALC and NLR, at the time of SRS actually respond more poorly to immunotherapy given later on (6). Given how widespread stereotactic radiation has become for treating patients with oligometastatic disease, the authors propose that identifying and targeting specific mechanisms of immunosuppression will be needed in the near future. Interestingly, some studies have instead shown that lymphopenia is a desirable outcome of chemoradiation; Sanchez-Perez et al. argue that the decrease in lymphocyte count due to chemotherapy causes a marked growth in the levels of various pro-inflammatory cytokines and a drop in the number of regulatory immune cells (7). The investigators describe how in both their preclinical mouse model as well as their Phase II GBM vaccine trial, diminishing the number of $\mathrm{T}$ cells actually augments the efficacy of immunotherapy and is associated with an increase in progression-free survival and overall survival.

As immunotherapeutic drugs influence the function of the immune system as a whole, not solely the lymphocyte cell line, it is important to look at immune system metrics beyond ALC and NLR. Although the interaction between neutrophils with cancer cells (and stromal cells within the tumor microenvironment) and the subsequent effects on tumor growth has been a distinct interest in preclinical and clinical models of cancer, many other pathways have been explored to provide potential candidate biomarkers for predicting tumor response. Much of the early work evaluating biomarkers of radiation exposure and response has been motivated by the need for radiation bio-dosimetry and countermeasure studies in radiation exposure scenarios including nuclear weapon detonation and dispersal of radioactive substances. Studies evaluating broad panels of biomarkers have focused on dose- and time-dependent changes in blood cell counts-markers of interest have included lymphocytes, neutrophils, platelets, red blood cells, NLR, wide panels of interleukins, granulocyte and granulocyte-macrophage colony stimulating factors, thrombopoietin, erythropoietin, acute-phase proteins such as amyloid A and LPS, and procalcitonin (8).

Along with the aggregate cell measures focusing on neutrophil and lymphocyte counts mentioned above, preoperative peripheral monocyte count greater than 300 
per cubic millimeter has been established as a prognostic biomarker in the setting of colorectal cancer with liver metastases (9). Studying the peripheral monocyte count as a biomarker was in part motivated by the known association of dendritic cells, derived from the peripheral monocyte component, with identification of antigen presenting cells and subsequent activation of antigen-specific cytotoxic lymphocytes. Modulation of monocyte-produced IL10 has also been studied as a biomarker of interest in the setting of metastatic melanoma (10). Instead of focusing on individual interleukins, cytokines, cell lines, or cell surface markers, additional studies have sought to use an aggregate inflammation score, calculated from a composite of other metrics. These include the Glasgow Prognostic Score, a graded score from 0 to 2 utilizing measures such as C-reactive protein and albumin as key determinants, which demonstrated seemingly independent predictive value for patient survival (beyond traditional staging and treatment covariates) in multiple studies across both operable and inoperable tumor types treated with chemotherapy and radiation (11). These markers have been effective insofar as they have highlighted associations with states of increased inflammation, though some have criticized them, as they do not highlight specific actionable targets (12).

Some studies have sought to further elucidate relationships under the broad category of surrogate biomarkers for evaluating the systemic immune response. The NLR, monocyte to lymphocyte ratio (MLR), and platelet to lymphocyte ratio (PLR) are commonly reported surrogates, though these measures do not often provide a complete story (13). The previously described NLR, for instance, can reflect a variety of conditions including underlying neutrophilia, lymphopenia, or both, and some investigators have sought to create a comprehensive profile of lymphocyte subpopulations. Such investigations have yielded associations such as high levels of CD4 tumor-infiltrating lymphocytes with low levels of CD8 tumor-infiltrating lymphocytes and worse prognosis in glioblastoma (14). Subsequent studies have also linked the Th $1^{\text {high }} \mathrm{Th} 2^{\text {low }}$ and Th $1^{\text {low }} \mathrm{Th} 2^{\text {low }}$ states with downregulation of PD-L1, PD-L2, and PD-1 (15). In the Th2 $2^{\text {low }}$ subgroup, fourteen genes were identified as potential prognostic markers. In this particular study, Takashima et al. express a hope that a balance of low Th2 activity with PD-L1 and PD-1 levels could be used to not only provide a molecular marker predictive of GBM survival but also identify associated genes serving as potential targets for immunotherapy.

Use of raw neutrophil and lymphocyte aggregate measures such as ALC and NLR are further complicated by the role of tumor microenvironment in changing the phenotype exhibited by altered levels of these lines. Neutrophils, in particular, have been described as exhibiting either an anti-tumoral (N1) or pro-tumoral (N2) phenotype depending on the tumor microenvironment $(14,16)$. Specifically, TLR, INF-gamma, TNF-a, IL-12 have been labeled as N1, anti-tumoral effectors, and NF-kB, STAT3, PI3k-AKT, IL-17/23, TGF-B, MMP-9, CXCR-2, NE, and HDAC determined to be N2, or pro-tumoral (12). With the recruitment of macrophages classified as part of the N1 neutrophil effector phenotype, tumor-associated macrophage count has been reported as a downstream biomarker of anti-tumoral systemic inflammatory response. Specifically, in the setting of non-muscle invasive bladder cancer, an inverse association was demonstrated between tumor-associated macrophage count and recurrence of carcinoma in situ following intravesical bacillus CalmetteGuérin therapy (17).

Therefore, Pike et al.'s recently published article surveying how palliative RT may lead to lower lymphocyte counts and therefore worse prognosis in the context of administering ICIs is an excellent example of how critical it is to remember that therapies are never given in a vacuum (1). One must consider the entire treatment paradigm as a whole in order to evaluate whether a treatment modality might augment or negate the efficacy of a second form of therapy. The authors' work is commended in attempting to address a timely and complex issue, as it is a valuable addition to the small yet growing body of literature that examines how to best manage patients to require treatment with both ICI and palliative RT.

However, the study is not without limitations; as with all retrospective studies, it may suffer from patient heterogeneity and unmeasured bias. For example, the authors observe that patients who received more than five fractions of RT were more likely to develop lymphopenia after completing radiation, but it remains unclear how much of this effect may be confounded by the malignancy itself. That is, patients who need more than five fractions of radiation likely have larger volume, bulkier metastases in more critical areas that may in and of itself affect immune system function. The heterogeneity in timing of blood draws in the study should also be noted, as the researchers allowed for variability of up to multiple weeks, relative to the radiation treatment itself, in scheduling each measurement of white blood cell count, which may have introduced additional uncertainty into their findings. Potential future 
studies that build on this work may include integrating measurements of immune system metrics into prospective, randomized trials investigating new immunotherapies in order to ascertain whether a differential response exists based on these laboratory markers. For example, it would be clinically relevant to determine whether new, practicechanging therapies such as durvalumab in Stage III nonsmall cell lung cancer have a less pronounced effect in those with severe lymphopenia (18). Finally, in the burgeoning field of immunotherapy, immune system function is commonly assessed through metrics like ALC and NLR, but looking at these values alone is likely insufficient. The importance of other laboratory markers such as levels of various interleukins and cytokines will need to be clarified in further research as potential prognostic factors.

\section{Acknowledgments}

Funding: None.

\section{Footnote}

Provenance and Peer Review: This article was commissioned and reviewed by the Section Editor Dr. Hsin-Hua Nien (Attending Physician, Department of Radiation Oncology, Cathay General Hospital, Taipei, Taiwan).

Conflicts of Interest: The authors have no conflicts of interest to declare.

Ethical Statement: The authors are accountable for all aspects of the work in ensuring that questions related to the accuracy or integrity of any part of the work are appropriately investigated and resolved.

Open Access Statement: This is an Open Access article distributed in accordance with the Creative Commons Attribution-NonCommercial-NoDerivs 4.0 International License (CC BY-NC-ND 4.0), which permits the noncommercial replication and distribution of the article with the strict proviso that no changes or edits are made and the original work is properly cited (including links to both the formal publication through the relevant DOI and the license). See: https://creativecommons.org/licenses/by-nc-nd/4.0/.

\section{References}

1. Pike LRG, Bang A, Mahal BA, et al. The Impact of
Radiation Therapy on Lymphocyte Count and Survival in Metastatic Cancer Patients Receiving PD-1 Immune Checkpoint Inhibitors. Int J Radiat Oncol Biol Phys 2019;103:142-51.

2. Grossman SA, Ye X, Lesser G, et al. Immunosuppression in patients with high-grade gliomas treated with radiation and temozolomide. Clin Cancer Res 2011;17:5473-80.

3. Su YB, Sohn S, Krown SE, et al. Selective CD4+ lymphopenia in melanoma patients treated with temozolomide: a toxicity with therapeutic implications. J Clin Oncol 2004;22:610-6.

4. Grossman SA, Ellsworth S, Campian J, et al. Survival in Patients With Severe Lymphopenia Following Treatment With Radiation and Chemotherapy for Newly Diagnosed Solid Tumors. J Natl Compr Canc Netw 2015;13:1225-31.

5. Fukunaga A, Miyamoto M, Cho Y, et al. CD8+ tumorinfiltrating lymphocytes together with CD4+ tumorinfiltrating lymphocytes and dendritic cells improve the prognosis of patients with pancreatic adenocarcinoma. Pancreas 2004;28:e26-31.

6. Li YD, Lamano JB, Kaur G, et al. Lymphopenia predicts response to stereotactic radiosurgery in lung cancer patients with brain metastases. J Neurooncol 2019;143:337-47.

7. Sanchez-Perez L, Suryadevara CM, Choi BD, et al. Leveraging chemotherapy-induced lymphopenia to potentiate cancer immunotherapy. Oncoimmunology 2014;3:e944054.

8. Ossetrova NI, Stanton P, Krasnopolsky K, et al. Biomarkers for Radiation Biodosimetry and Injury Assessment after Mixed-field (Neutron and Gamma) Radiation in the Mouse Total-body Irradiation Model. Health Phys 2018. [Epub ahead of print].

9. Sasaki A, Kai S, Endo Y, et al. Prognostic value of preoperative peripheral blood monocyte count in patients with colorectal liver metastasis after liver resection. J Gastrointest Surg 2007;11:596-602.

10. Torisu-Itakura H, Lee JH, Huynh Y, et al. Monocytederived IL-10 expression predicts prognosis of stage IV melanoma patients. J Immunother 2007;30:831-8.

11. McMillan DC. The systemic inflammation-based Glasgow Prognostic Score: a decade of experience in patients with cancer. Cancer Treat Rev 2013;39:534-40.

12. Schernberg A, Blanchard P, Chargari C, et al. Neutrophils, a candidate biomarker and target for radiation therapy? Acta Oncol 2017;56:1522-30.

13. Song S, Li C1, Li S, et al. Derived neutrophil to 
lymphocyte ratio and monocyte to lymphocyte ratio may be better biomarkers for predicting overall survival of patients with advanced gastric cancer. Onco Targets Ther 2017;10:3145-54.

14. Han S, Zhang C, Li Q, et al. Tumour-infiltrating CD4(+) and CD8(+) lymphocytes as predictors of clinical outcome in glioma. Br J Cancer 2014;110:2560-8.

15. Takashima Y, Kawaguchi A, Kanayama T, et al. Correlation between lower balance of Th2 helper T-cells and expression of PD-L1/PD-1 axis genes enables prognostic prediction in patients with glioblastoma. Oncotarget

doi: 10.21037/tro.2019.09.01

Cite this article as: Chen VE, Greenberger BA, Taylor JM, Lu B. Key immune system markers in immunotherapy: absolute lymphocyte count, neutrophil to lymphocyte ratio, and alternative immune system metrics. Ther Radiol Oncol 2019;3:33.
2018;9:19065-78.

16. Fridlender ZG, Sun J, Kim S, et al. Polarization of tumorassociated neutrophil phenotype by TGF-beta: "N1" versus "N2" TAN. Cancer Cell 2009;16:183-94.

17. Ajili F, Kourda N, Darouiche A, et al. Prognostic value of tumor-associated macrophages count in human non-muscle-invasive bladder cancer treated by BCG immunotherapy. Ultrastruct Pathol 2013;37:56-61.

18. Antonia SJ, Villegas A, Daniel D, et al. Durvalumab after Chemoradiotherapy in Stage III Non-Small-Cell Lung Cancer. N Engl J Med 2017;377:1919-29. 